\title{
"Hybrid" Microscopy: Multimodal, Correlative and Dynamic Characterization of Soft and Hybrid Structures
}

\author{
Vinayak P Dravid ${ }^{1,2 *}$ \\ 1. Department of Materials Science \& Engineering, Northwestern University, Evanston, IL, USA. \\ 2. The NUANCE Center, Northwestern University, Evanston, IL, USA. \\ * Corresponding author: v-dravid@northwestern.edu
}

There have been remarkable developments in materials in recent couple decades with the inception of nanoscale synthesis and architecturing of hard, soft and hard-soft hybrid systems. The latter materials, in particular, present special challenges for characterization and analysis. This is due to the diverse and often diametrically opposite considerations for microscopy and analysis. The "soft" side of the structure mandates low dose mode in microscopy and need for contrast enhancement through staining and related approaches. On the other hand, the "hard" part of the hybrid systems are often robust enough for radiation abuse in microscopy and analysis. These contraindicating considerations make "soft" and "hybrid" microscopy particularly challenging.

At the NUANCE Center of Northwestern University, we have been advancing multimodal, correlated and dynamic characterization of soft and hybrid systems. The soft-hard hybrid systems that are being developed are diverse, and encompass classical soft-hard interfaces such as biofilms on hard surfaces or polymer-nanoparticle composites to more exotic ones like DNA-assembled nanoparticles or Covalent/Metal-Organic Framework (COF/MOF) structures and composites. To mitigate the contrast issues in soft part of the hybrid systems, we invoke the modification of the so-called ChromEMT [1] approach for enhancing contrast of macromolecular structures. The nominal ChromEMT approach is now being extended to other cellular and non-cellular systems to enhance contrast, while sparse and dynamic sampling further reduce the deleterious electron flux which otherwise compromises the sample stability and integrity. The recent addition of high performance direct electron detectors (e.g., so-called K2/K3 cameras) at NUANCE provides additional benefit of sensitivity, thereby helping further reduce electron flux for a given contrast threshold. Further, the microscopy efforts are complemented by correlative characterization, typically with optical, confocal/fluorescence and related measurements. In some cases, the complementarity is achieved through synchrotron $\mathrm{x}$-ray scattering that provide reciprocal information which may need real-space modeling through microscopy.

The presentation will cover several examples of this complex choreography of multimodal and correlative characterization of soft and soft-hard hybrid systems. Figure 1 illustrates the various facets of our approach and methodologies, along with illustrative thumbnail examples of soft and hybrid systems. For example, in case of complex architecture comprising DNA-bound nanoparticles [2], we show that the DNA backbone can be used to anchor silica precursor to convert into a rigid framework for subsequent microscopy and tomography. More recently, it is reported that using silver ion intercalation of DNA greatly enhances stability that renders the assembled structure conducive for electron microscopy/analysis. On the sparse and dynamic sampling for imaging and analysis [3], we show that it is possible to incorporate sampling approach to minimize electron flux for spectroscopy and spectroscopic imaging yet achieve the fidelity necessary to discriminate chemical partitioning with EDS and EELS. Finally, the availability of direct electron detectors have truly transformed imaging and analysis of other soft and hybrid structures such as COFs and MOFs; in addition to obvious advantages for in-situ microscopy. The 
presentation will cover emerging examples of soft materials utilizing the multimodal, correlative and dynamic characterization [4].

\section{References:}

[1] HD Ou et al., Science 357 (2017), p. eaag0025.

[2] Q Li et al., Science (2018). DOI:10.1126/science.aaq0591

[3] K Hujsak et al., Micron 108 (2018), p. 31.

[4] This work is based on research sponsored by the Air Force Research Laboratory under agreement No. FA8650-15-2-5518, Air Force Office of Scientific Research under Award No. FA9550-12-10280. This work made use of the EPIC facility of Northwestern University's NUANCE Center, which has received support from the Soft and Hybrid Nanotechnology Experimental (SHyNE) Resource (NSF ECCS-1542205); the MRSEC program (NSF DMR-1720139) at the Materials Research Center; the International Institute for Nanotechnology (IIN); the Keck Foundation; and the State of Illinois.

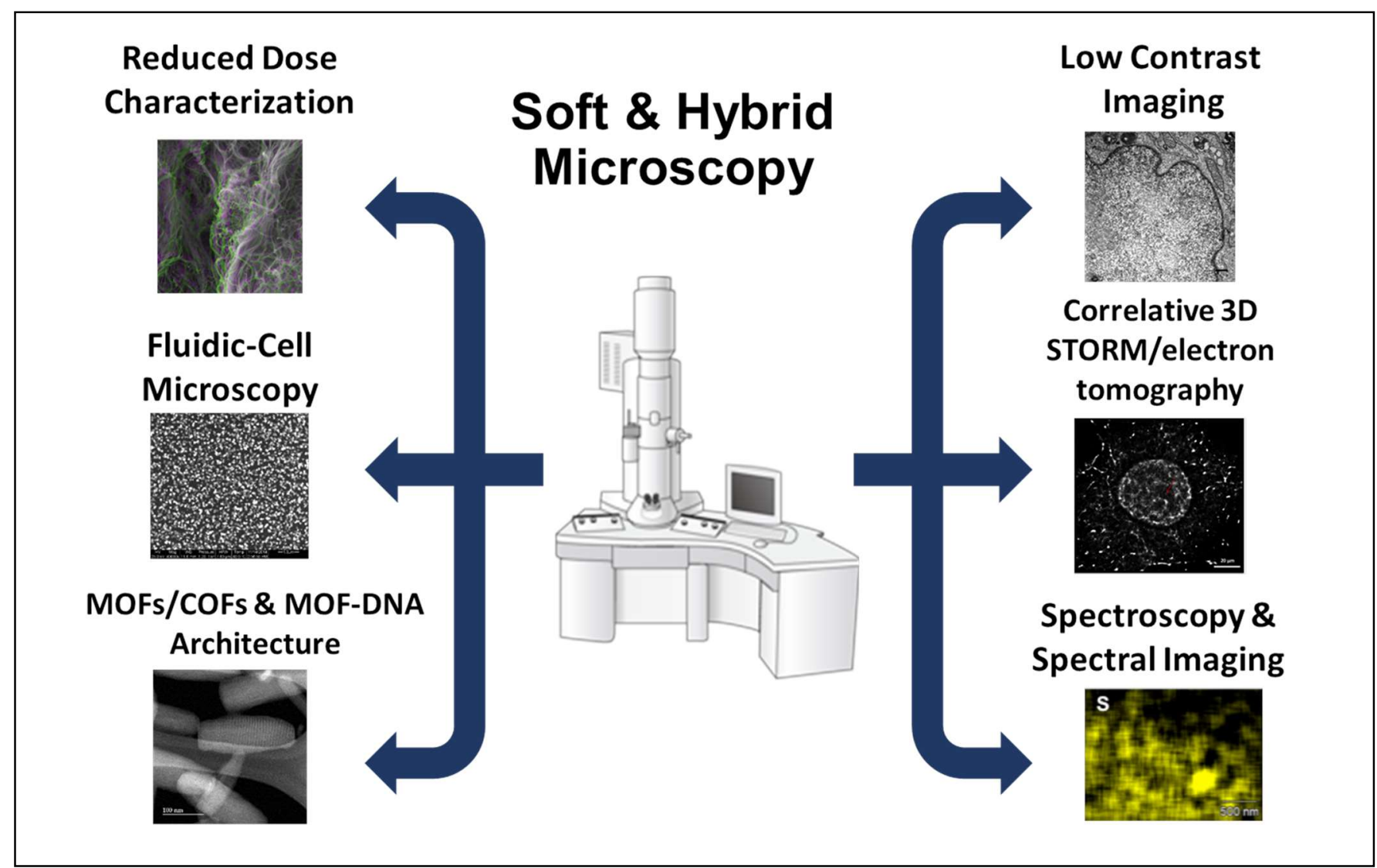

Figure 1. Illustration of various soft and hybrid microscopy and analysis approaches undertaken at the NUANCE Center for soft and hybrid structures and systems. 